
\title{
$\angle S$ Research Square \\ Selection Stability in High Dimensional Statistical Modelling: Defining a Threshold for Robust Model Inference
}

Martin Green ( $\sim$ martin.green@nottingham.ac.uk)

University of Nottingham

Eliana Lima

European Food Safety Authority

Robert Hyde

University of Nottingham

\section{Research Article}

Keywords: Covariate selection, selection stability, stability threshold, high dimensional data, statistical triangulation.

Posted Date: August 2nd, 2021

DOI: https://doi.org/10.21203/rs.3.rs-738092/v1

License: (a) (1) This work is licensed under a Creative Commons Attribution 4.0 International License. Read Full License 


\section{Title}

2 Selection stability in high dimensional statistical modelling: Defining a threshold for robust

3 model inference

4

\section{Author names and affiliations}

6 Martin Green ${ }^{\mathrm{a}^{*}}$, Eliana Lima ${ }^{\mathrm{a}, \mathrm{b}}$, Robert Hyde

7

$8{ }^{a}$ School of Veterinary Medicine and Science, University of Nottingham, Sutton Bonington Campus,

9 Leicestershire, United Kingdom

$10{ }^{\mathrm{b}}$ Present address: European Food Safety Authority, Via Carlo Magno 1A, 43126, Parma, Italy

11

12 *Corresponding author

13 Martin Green

14 E-mail: martin.green@nottingham.ac.uk

15 School of Veterinary Medicine and Science, University of Nottingham, Sutton Bonington Campus,

16 Leicestershire, LE12 5RD, United Kingdom

$17 \quad$ Tel +44 1159516116

18

19

20 Declarations of interest: none

21 


\section{Abstract}

23 Epidemiological research commonly involves identification of causal factors from within

24 high dimensional (wide) data, where predictor variables outnumber observations. In this

25 situation, however, conventional stepwise selection procedures perform poorly. Selection

26 stability is one method to aid robust variable selection, by refitting a model to repeated

27 resamples of the data and calculating the proportion of times each covariate is selected. A key

28 problem when applying selection stability is to determine a threshold of stability above which

29 a covariate is deemed 'important'.

30 In this research we describe and illustrate a two-step process to implement a stability

31 threshold for covariate selection. Firstly, covariate stability distributions were established

32 with a permuted model (randomly reordering the outcome to sever the relationship with

33 predictors) using a cumulative distribution function. Subsequently, covariate stability was

34 estimated using the true model outcome and covariates with a stability above a threshold

35 defined from the permuted model, were selected in a final model. The proposed method

36 performed well across 22 varied, simulated datasets with known outcomes; selection error

37 rates were consistently lower than conventional implementation of equivalent models. This

38 method of covariate selection appears to offer substantial advantages over current methods, to

39 accurately identify the correct covariates from within a large, complex parameter space.

\section{Keywords}

44 Covariate selection; selection stability; stability threshold; high dimensional data; statistical 45 triangulation. 


\section{Introduction}

48 Epidemiological research increasingly involves identification of potentially causal factors

49 from within relatively wide datasets, that is, when the number of variables (p) is relatively

50 large compared to the number of observations (n). Identification of causal variables for

51 inference from within a high dimensional, wide dataspace is problematic because conventional stepwise selection procedures perform poorly, resulting in over fit models ${ }^{1-4}$.

Statistical methods have been developed to improve variable selection with such data, including modifications to $\mathrm{AIC} / \mathrm{BIC}^{5}$, and a range of regularisation methods based on functions that penalise model coefficients to balance over and under fitting, the so called variance-bias trade off ${ }^{6-8}$.

In addition to regularisation, it is acknowledged that robustness in model selection is improved through use of covariate selection stability ${ }^{9-11}$. Covariate stability is estimated by refitting a model to repeated resamples of the data (for example through Bootstrapping) and

60 calculating the proportion of times each covariate is selected across all resamples. Whilst it is

61 known that the most stable variables (those selected in most subsampled models) are least

62 likely to be false positives ${ }^{9}$, the optimal threshold for stability, above which a covariate should be deemed 'important' or 'significant', has not been determined. A stability threshold originally proposed for use with lasso regression ${ }^{9}$ has been shown to be too conservative, resulting in true causal variables being missed ${ }^{12,13}$. An empirical method of stability selection, proposed for genetic data using elastic net regression ${ }^{13}$, appeared to improve upon the method proposed by Meinshausen and Bühlmann ${ }^{9}$, but the issue of missing many true causal variables (false negative results) remained. To date, since a clear, generalisable method to identify a stability threshold is unavailable, arbitrary thresholds have been employed for stability analyses in veterinary epidemiology ${ }^{14,15}$ and the need for research to 
establish a suitable cut-off for the covariate selection in high dimensional data is clear and has been recently re-emphasised in human epidemiology ${ }^{16}$.

73 In this paper we build on principles described by Meinshausen and Bühlmann ${ }^{9}$ and Kim et al ${ }^{13}$, to develop a general approach to identify a threshold for covariate selection stability and hence to aid robust model selection for high dimensional, correlated data. To evaluate and illustrate the method, we use 22 simulated datasets with known outcomes and increasingly complex correlation structures, alongside five methods of covariate selection to illustrate generalisability. For each statistical method we compare the performance of covariate selection based on a conventional approach to that using the application of selection stability with the newly defined threshold.

81

\section{Materials and Methods}

Five statistical methods were used to analyse 22 simulated datasets that were constructed with known underlying relationships and correlation structures. For each method, a conventional approach to covariate selection was conducted followed by implementation of selection stability by bootstrapping ${ }^{14}$. The modelling methods chosen are acknowledged approaches for inferential modelling and were stepwise selection based on Akaike information criterion (sAIC) ${ }^{17}$, stepwise selection based on a modified Bayesian Information Criterion (mBIC) ${ }^{5}$, elastic net regression (enet) ${ }^{8}$, minimax convex penalty regression (MCP) ${ }^{18}$ and a combination method that synthesised results from all four methods ${ }^{19}$. These modelling techniques are described in detail in Section 2.1 and the new approach to stability selection, including the determination of a threshold for inference, is described in Section 2.2.

93 All datasets used for analysis are described in Section 2.3. 


9

The following statistical approaches were used to evaluate and compare covariate selection.

A conventional linear regression model was implemented using the 'stepwise' function in the bigstep package ${ }^{20}$ in $\mathrm{R}^{21}$. The regression equation took the form;

$$
y=\beta_{0}+\sum_{j=1}^{p} \beta_{j} x_{j}+e
$$

where $y$ was the response variable specified in the simulated data, $\beta_{0}$ an intercept term, $x_{j}$ represented the $\mathrm{j}^{\text {th }}$ of $p$ covariates with an estimated coefficient $\beta_{j}$ and $e$ the residual model error. To speed computation, variable selection was conducted by first removing explanatory variables with a relatively poor correlation with the outcome (Pearson correlation, $\mathrm{P}>0.05$ ) followed by an iterative stepwise procedure (forward and backward) with selection of the final set of variables through minimisation of the Akaike information criterion (AIC). The AIC loss function is defined as; $2 \mathrm{k}-2 \ln (\hat{\mathrm{L}})$, where $\mathrm{k}$ is the number of parameters in the model and $\hat{L}$ the likelihood function.

A modified Bayesian Information Criterion was used for model selection, also implemented in the bigstep package ${ }^{20}$ with the 'stepwise' function being used for covariate selection. The modified BIC increases the penalty on the number of parameters selected beyond the conventional BIC, producing a sparser model. The mBIC loss function was implemented as;

$$
m B I C=\log L\left(Y \mid M_{i}, \theta_{i}\right)-\frac{1}{2} k_{i} \log n-k_{i} \log \left(\frac{1-p}{p}\right)
$$

18 where $\log L\left(Y \mid M_{i}, \theta_{i}\right)$ represented the $\log$ likelihood given model $M_{i}$ and parameter 9 values $\theta_{i}, k_{i}$ was the number of predictors in the selected model, $n$ the sample size, and $p$ 
the probability that a randomly chosen predictor influenced Y. As the number of available predictors increases relative to the number of samples (n), p decreases and $k_{i} \log \left(\frac{1-p}{p}\right)$ becomes of increasing importance as the penalty term ${ }^{5}$.

Elastic net is a form of regularised regression that incorporates a mixture of lasso (L1) and ridge (L2) penalties ${ }^{8}$ and was implemented as;

$$
S S E_{\text {enet }}=\frac{1}{2 n} \sum_{i=1}^{n}\left(y_{i}-\hat{y}_{i}\right)^{2}+\lambda_{E}\left[\sum_{j=1}^{P} \frac{1}{2}(1-\alpha) \beta_{j}^{2}+\alpha\left|\beta_{j}\right|\right]
$$
where $S S E_{\text {enet }}$ represented the elastic net loss function to be minimised, i denoted each observation and $\mathrm{n}$ the sample size, $y_{i}$ and $\hat{y}_{\mathrm{i}}$ were the simulated true and model predicted outcomes respectively for the ith observation, $j$ denoted a predictor variable with $\mathrm{p}$ the number of predictor variables in total, and $|\beta|$ represented absolute values of the regression coefficients. The hyperparameters that provide the penalty $\left(\lambda_{E}\right)$ and the relative proportion of penalisation on either the sum of the square of the coefficients or the unsquared coefficients $(\alpha)$ were optimised using $10 \times 10$-fold cross validation to minimise mean absolute error (MAE). Elastic net models were built using the glmnet package ${ }^{22}$ using the caret package platform ${ }^{23}$ in $\mathrm{R}^{21}$.

$$
S S E_{m c p}=\sum_{i=1}^{n}\left(y_{i}-\hat{y}_{\mathrm{i}}\right)^{2}+\sum_{j=1}^{p} P\left(\beta_{j} \mid \lambda, \gamma\right)
$$


145 where SSEmcp represented the loss function to be minimised, $i, y_{i}, \hat{y}_{\mathrm{i}}, j, p$ and $n$ were as 146 defined in equation (3) and $P\left(\beta_{j} \mid \lambda, \gamma\right)$ represented a penalty function as follows; where $\gamma$ and $\lambda$ were hyperparameters optimised using $10 \times 10$-fold cross validation to minimise the MAE. MCP models were estimated using the R package ncvreg ${ }^{24}$.

155 Conventional bootstrapping ${ }^{25}$ was used to estimate covariate stability for all analytic approaches, according to methods previously described ${ }^{26}$. In brief, selection stability ${ }^{9,10,27}$

157 was evaluated for each model as the percentage of times each covariate was selected across

158200 bootstrap samples. For elastic net and MCP, model hyperparameters used in the

159 bootstrap sampling were those identified using $10 \times 10$-fold cross validation in an initial

160 model using the full dataset. The distributions of variable coefficients were calculated from

161 all non-zero values of the coefficient in the bootstrap samples and a bootstrap P value (BPV)

162 was calculated for each covariate ${ }^{14}$. The BPV was calculated from all non-zero coefficient

163 values of the bootstrap sample (i.e. when the variable was selected in the model) and defined

164 as the smallest proportion of (non-zero) coefficient values to one side of zero. For example, if

165 a covariate was selected in the model in 100 bootstrap samples and 95 of these were either

166 greater or less than zero, then the Bootstrap P value would be; $(100-95) / 100=0.05$. Variable

167 selection was visualised by plotting covariate selection stability against Bootstrap P value. 
170 Covariate selection stabilities were combined across all modelling methods using an

171 approach described previously ${ }^{19}$. In brief, the bootstrap matrices from each individual

172 modelling method were aligned by covariate to create a single matrix containing an equal

173 number of bootstrap samples from each method. This combined matrix was used to calculate

174 overall covariate selection stabilities and Bootstrap P values as described above. Therefore

175 this method provided an estimate for selection stability and Bootstrap P values for all

176 covariates synthesised across all four methods of covariate selection.

177

178

179

180

181

182

183

184

185

186

\subsection{Stability threshold calculation}

A stability threshold above which each covariate was deemed to be important and selected in a final model ('selection stability threshold $\left(\mathrm{T}^{\mathrm{S}}\right)$ '), was calculated as follows. The concept was to initially determine a distribution of covariate stability for each dataset on the basis that there was no casual relationship between the outcome and explanatory variables and to compare this to the stability of covariates in the actual data. The distribution of covariate stability when no causal relationship existed we name here, the 'baseline stability distribution'. When the stability of a covariate in the actual data had a specified probability of exceeding values in the baseline stability distribution, it was deemed to be important and selected in the final model.

For each model and dataset, the baseline stability distribution was determined by randomly permuting the outcome variable to sever any relationship between outcome and explanatory variables. Using the permuted outcome, a stability analysis was conducted as described in Section 2.2 and the baseline stability of all covariates estimated. An empirical cumulative distribution function (ECDF) of the baseline stability distribution was formulated and 
193 stability thresholds were tested at different specified probability values of the ECDF. The

194 specified probability values were calculated as;

196 where $\mathrm{x}$ was either equal to $0,1,3,5$, or 10 and $\mathrm{p}$ the number of covariates available in a

197 dataset. For example, when $\mathrm{x}=1$ and 500 potential covariates were available in the dataset,

198 the probability value of the ECDF at which the stability threshold would be set was;

$$
1-(1 / 500)=0.998
$$

200 Therefore, $x / p$ represented the probability that the stability of any covariate would exceed the 201 defined stability threshold.

203 To clarify the method, we list the steps used to calculate $\mathrm{T}^{\mathrm{S}}$;

204 i. For a specified dataset and model, the outcome variable was randomly permuted to remove the relationship between the outcome and explanatory variables.

ii. Covariate selection stability was conducted (as described in Section 2.2) using a set of 20 bootstrap samples.

iii. The distribution of covariate stability values for all ' $p$ ' covariates was defined using an empirical cumulative distribution function ('ecdf' function, base R) and the (1-x/p) probability value of the distribution calculated, to provide the stability threshold $\mathrm{T}^{\mathrm{S}}$. The values tested for ' $\mathrm{x}$ ' were $0,1,3,5$ and 10 .

212 iv. Steps i. to iii. were repeated ten times and the mean and standard error for $\mathrm{T}^{\mathrm{S}}$ 213 calculated across the ten repeats.

214 v. Covariate selection stability was conducted on the full dataset (i.e. without permuting the outcome variable) using 200 bootstrap samples and the mean value of $\mathrm{T}^{\mathrm{S}}$, as calculated in steps $\mathrm{i}$ - iv, used as the threshold above which variables were considered to be 'true' and therefore selected in the final model. 
218 Therefore, the stability threshold was set at a value at which there was a low probability (x/p)

219 of a covariate reaching this level of stability if it was not truly associated with the outcome.

220 Importantly, since location of the threshold is likely to be dependent upon the method of

221 modelling and specific dataset used, a new stability threshold was calculated for each dataset

222 and analysis undertaken.

223

2.5. Simulated datasets

225

The purpose of simulating data was to construct a set of realistic datasets, of varying size and complexity, in which true underlying causal relationships were known. Therefore, model performance could be evaluated objectively by comparing the accuracy with which each method (including the stability threshold method) correctly selected the true causal variables. For each dataset, 8 covariates were used to calculate an outcome variable; we refer to these as the 'true' covariates. Additional noise variables were generated for each dataset, randomly and independent of the outcome, we refer to these as 'false' variables. In each dataset, true and false variables were set to be correlated with each other to a different extent such that each statistical method could be evaluated with different but realistic complexity added to the data (Table 1).

In all datasets, an outcome variable, “y_out”, was calculated from the true explanatory variables as follows;

$$
y_{-} \text {out }=\text { Intercept }+\sum_{k=1}^{r} 2.5 x_{k}+\sum_{j=1}^{q} 2.5 x_{j}+e
$$

where Intercept $=1, x_{k}$ represented the $\mathrm{k}^{\text {th }}$ of $r$ true covariates simulated with no specified correlation with other covariates, $x_{j}$ represented the $\mathrm{j}^{\text {th }}$ of $q$ true covariates that were specified

242 to have correlations with a set of ten false explanatory covariates, and $e$ was a random 
243 variable that represented all other true but unknown effects that causally influenced the

244 outcome, $y_{-}$out. $e$ was drawn from a Normal distribution with mean $=0$ and standard

245 deviation which varied between dataset to allow the amount of variability explained by the

246 true covariates to differ between datasets (Table 1).

247 All explanatory covariates were drawn from distributions with mean $=0, \mathrm{SD}=1$; they

248 represented standardised variables. Covariates that were simulated to be correlated $\left(x_{j}\right)$ were

249 drawn from a multivariate normal distribution (using the mvrnorm function in the MASS

250 package ${ }^{28}$ in $\left.\mathrm{R}\right)$. In this case, each variable drawn from a distribution with mean $=0, \mathrm{SD}=1$

251 and with covariance matrix specified such that variables were generated with a correlation

252 between $0.3-0.7$, depending on the dataset (Table 1 ).

253 The characteristics of all datasets are summarised in Table 1. For each dataset, conventional

254 linear regression was used to model solely the true covariates, to illustrate the underlying

255 relationship between the outcome and true variables and estimate the total amount of

256 variation explained. True variables were simulated in all datasets such that their partial

257 coefficients in these regression models were individually significant $(\mathrm{P}<0.05)$; that is their

258 signal was sufficiently strong to be deemed 'significant' when modelled alone. The purpose

259 of datasets containing more highly correlated covariates was to evaluate the extent to which

260 true variables could be retrieved when a substantial quantity of random and correlated noise

261 was added to the data and to explore whether selection stability enhanced retrieval.

262 For four of the 22 generated datasets a binary outcome was simulated instead of a continuous

263 outcome so that the analytic methods could be evaluated using an alternative outcome

264 distribution. A similar approach was used as in equation (6) except that an inverse logit

265 function followed by a draw from a binomial distribution was used to alter the outcome to be

2661 or 0 . As for the datasets with normally distributed outcomes, correlations were included

267 between true and false explanatory covariates to provide an increasing complexity within the 
268 data. The variation explained by the true variables was estimated using an adapted $\mathrm{R}^{2} 29$;

269 further details of the binomial outcome datasets are provided in Table 1.

270

$271 \quad$ Table 1 approx here

272

273

2.6. Evaluation and comparison of model performance

Model performance was assessed based on the correct selection of true variables and correct omission of the false variables, and comparisons were made between methods for all datasets.

Graphical analysis was used to visualise model outcomes; scatterplots were used to illustrate results of the stability analysis by plotting the stability (\%) against BPV for covariates selected in the model, as previously reported ${ }^{19}$ but with the additional annotation of the selection stability threshold.

\section{Results}

\subsection{Error rates for full (non-bootstrapped) models}

A summary of the performance of all full (non-stability threshold) modelling methods, for each dataset is provided in Table 2. Across all datasets, model selection using sAIC, elastic net regression and MCP included a higher number of incorrect (false positive and false negative) variables than selection using mBIC. The incorrect covariates selected using sAIC, enet and MCP were mostly false positives indicating these models generally over fit the data. Whilst the final models produced using mBIC were more sparse than for other methods (fewer variables selected in the final models), the incorrectly classified covariates were mostly false negatives indicating a tendency towards under fitting with this method. In general, for most methods, more covariates were incorrectly classified as the complexity of correlation structure within the data increased (Table 2). 
294 \{Table 2 approx here please\}

295

296

3.2. Error rates for models incorporating covariate selection stability

297 Results of the covariate selection stability analyses using stability thresholds calculated with

$298 \mathrm{x}=0$, are provided in Table 3. Results for all other thresholds, $\mathrm{x}=1,3,5$ and 10, are

299 provided in Supplementary Materials (Tables S1-S6). The stability thresholds that produced

300 fewest incorrectly classified covariates (false positives and false negatives) across all models

301 and all datasets were those calculated using $\mathrm{x}=0$ or $\mathrm{x}=1$ but the threshold calculated with $\mathrm{x}$

$302=0$ selected fewest false positive covariates and was therefore judged the optimal threshold to

303 use. As expected, with increasing values of $\mathrm{x}$, the number of false positive variables selected

304 tended to increase and the number of false negatives decreased. The one exception to $\mathrm{x}=0$

305 being the optimal threshold was the highly complex dataset IC2, in which the stability

306 threshold at $x=3$ produced very slightly better results than $x=0$ or $x=1$ (Table $S 6$ ).

307 Importantly, for all modelling methods, use of bootstrap selection stability at a threshold

308 using $\mathrm{x}=0$ resulted in substantially fewer covariates being incorrectly selected (Table 3 )

309 compared to the conventional implementation of each technique (Table 2). For sAIC, enet

310 and MCP the improvement in accuracy of covariate selection was marked; the number of

311 false positive covariates was greatly reduced with no increase in false negatives. For mBIC

312 the improvement in performance was less marked but still evident. The combination method,

313 a synthesis of methods incorporating results from all four model types, produced the best

314 overall performance compared to other methods, with the lowest number of false positive

315 variables selected whilst maintaining a low number of false negative covariates.

$317 \quad$ Table 3 approx here 
319 Across all model types, it was noticeable that as complexity of correlations within the

320 simulated data increased, accuracy of covariate selection slightly deteriorated. Importantly, in

321 the datasets that were simulated with no or relatively few underlying correlations (Datasets

322 A-C), with a selection stability threshold set at $\mathrm{x}=0$ or $\mathrm{x}=1$, all model methods achieved

323 near-perfect selection accuracy when stability selection was used (Table 3). This was in

324 contrast to the performance of the models without selection stability (Table 2) in which only

325 mBIC models performed well on non-correlated data. As the size and number of correlations

326 within the data increased, performance of all methods slightly deteriorated, however, model

327 performance remained much better with the use of covariate selection stability than for

328 conventional implementation of each model type.

330 3.3. Diagrammatic illustration of model performance

331 Covariate selection stability thresholds for all values of $\mathrm{x}$ are illustrated graphically for

332 datasets MS1, MS2 and LS4, in Figures 1 - 3 respectively. The graphs provide an illustration

333 of the positioning of the stability thresholds $\left(\mathrm{T}^{\mathrm{S}}\right)$ relative to the known true and false

334 covariates, for different values of $\mathrm{x}$. Use of 10 replicates of 20 sets of bootstrap samples was

335 sufficient to produce relatively small $95 \%$ confidence intervals for $\mathrm{T}^{\mathrm{S}}$ although the number of

336 replicates can be increased to further reduce the confidence interval as required.

$338 \quad$ \{Figure 1 here, to be reproduced in colour please

Figure 1. Graphical representation of model results from dataset MS1. Each scatterplot 
$344(M C P)$ and the four methods combined (combi). The y-axis defines the negative bootstrap P value 345 for each covariate (-BPV) and the $x$-axis represents covariate stability (\%). The dashed lines on 346 each graph (from right to left) represent the stability threshold calculated at $x=0,1,3,5$ and 10

347 respectively. The shaded grey area depicts the $95 \%$ confidence interval for the stability threshold $348 \quad$ calculated at $x=0$.

$350 \quad$ \{Figure 2 here please, to be reproduced in colour please\} illustrates results of the stability analysis for one of five statistical methods; stepwise selection using Akaike information criterion (AIC), stepwise selection using a modified Bayesian Information Criterion (mBIC), elastic net regression (enet), Minimax Convex Penalty regression $(M C P)$ and the four methods combined (combi). The y-axis defines the negative bootstrap P value for each covariate (-BPV) and the $x$-axis represents covariate stability (\%). The dashed lines on each graph (from right to left) represent the stability threshold calculated at $x=0,1,3,5$ and 10 respectively. The shaded grey area depicts the $95 \%$ confidence interval for the stability threshold \{Figure 3 here, to be reproduced in colour please calculated at $x=0$. results of the stability analysis for one of five statistical methods; stepwise selection using Akaike information criterion (AIC), stepwise selection using a modified Bayesian Information Criterion (mBIC), elastic net regression (enet), Minimax Convex Penalty regression (MCP) and the four methods combined (combi). The y-axis defines the negative bootstrap P value for each covariate 
$(-B P V)$ and the $x$-axis represents covariate stability (\%). The dashed lines on each graph (from right to left) represent the stability threshold calculated at $x=0,1,3,5$ and 10 respectively. The

\section{Discussion}

375 Our results using simulated datasets with known relationships highlight the value of selection stability as a means to identify the correct explanatory variables when the number of covariates $(p)$ is relatively large with respect to the number of observations $(n)$. Whilst the concept of selection stability is not new ${ }^{9,27}$, its use within epidemiological fields has been relatively limited. It is generally considered that selection stability is a necessary and beneficial approach to enhance inferential modelling with high dimensional data ${ }^{11,30}$ and that

381 without selection stability results of different selection methods vary dramatically leading to confusion in inference ${ }^{26,31}$.

383 An important consideration, however, when employing selection stability is to identify a

384 stability threshold above which covariates are deemed 'important' or 'significant' and

385 selected in a final model; this was the primary purpose of the current study. Whilst it is

386 known that a higher threshold for selection stability will result in the selection of fewer false

387 positive covariates ${ }^{9}$, a generalisable approach to identify an optimal threshold suitable for

388 multiple model types has not been reported. A threshold proposed for one model type, lasso

389 regression ${ }^{9}$ has been shown to be too conservative with too few true variables being selected

$390 \quad 13,32$. A further problem with the threshold proposed by Meinshausen and Bühlmann ${ }^{9}$ is that it

391 cannot be defined at a value $<50 \%$ which, as we have shown in this research, is certainly

392 necessary with some types of models and data (Figures 1-3). 
393 In this study we defined and evaluated a straightforward approach to identify a covariate

394 selection stability threshold that can be readily used with different datasets and model types.

395 The intuitive approach of using an expected or 'baseline' stability distribution from a model

396 with no underlying relationships and using the same data to compare this to a distribution

397 from a model with causal relationships, worked well across all five model types and all 22

398 complex datasets. Even for the highly correlated datasets, error rates were relatively low and,

399 importantly, were markedly superior to the equivalent conventional (non-stability selection)

400 models. Therefore, for safety of inference, when using relatively wide, high dimensional

401 datasets, covariate selection stability with the defined threshold will minimise identification

402 of false positive and false negative variables compared to conventional modelling techniques.

403 Furthermore, although highly correlated data are generally recognised to distort model

404 parameters ${ }^{33}$, our results indicate that selection stability with an appropriate choice of

405 threshold provides a method to mitigate this issue.

406 The concept of permuting variables to enhance statistical inference is not new. It has been

407 used in machine learning to facilitate estimation of variable importance ${ }^{34,35}$ and as a

408 replacement for cross validation to identify covariates in elastic net regression using non-

409 sparse genetic data ${ }^{13}$. In the setting we describe, permuting the outcome allowed

410 identification of an optimal covariate stability threshold to identify true variables for a variety

411 of modelling methods and types of data. Whilst using covariate selection stability was more

412 computationally expensive than conventional approaches, this was not prohibitive. For

413 example, for a set of 10 repeats of 20 bootstrap samples (to define the baseline stability

414 threshold) followed by 200 bootstrap samples (to select variables above the baseline

415 threshold) using a dataset with 500 observations and 1000 covariates, the total time taken for

416 computation of the mBIC method was 3 minutes and 16 seconds using a 15 core i9 processor

417 with $32 \mathrm{~GB}$ RAM. The equivalent time for the elastic net model was 28 minutes and 4 
418 seconds. In terms of the substantial improvements in accuracy of covariate selection, the

419 additional time required would appear to be very worthwhile.

420 Also worthy of note in our results was the excellent performance of the combination

421 modelling method; this is in agreement with previous research ${ }^{19}$. For all datasets this method

422 provided the most accurate variable selection and this is likely to be due to the principle of

423 triangulation. Triangulation is based on the concept that reliability of results is enhanced

424 through integration of different approaches, particularly when each approach has a different,

425 unrelated source of bias ${ }^{36}$. For this reason, it has been suggested that triangulation should be

426 more widely adopted to ensure robustness and reproducibility of scientific results ${ }^{37}$ although

427 it has rarely been employed in animal health research.

4.1. Study limitations

There were several limitations of this study worthy of consideration. Although substantial complexity was incorporated into the simulated datasets, no clustering of the outcome variable was considered. Therefore, extension of this stability threshold method to mixed effect models, although likely to be valid, requires additional investigation. Similarly, nonlinearities within predictor variables were not considered in the simulated data and this also warrants additional exploration in terms of selection stability thresholds. Four methods of modelling were evaluated, and a combination of all four together, which suggests our proposed method of selection stability may be generalisable, however, further research using additional model types would be worthwhile. In addition, although one group of simulated dataset incorporated discrete (binary) outcome variables (Datasets BIN1-4), further research would be valuable to investigate the value of these methods with outcome distributions of

441 varying types. Finally, it should be noted that underlying true solutions in our simulated data 442 were relatively sparse; eight covariates were chosen to have true causal influences. This 
443 aligns with the 'bet on sparsity principle' ${ }^{4}$, which dictates that for causal variables to be

444 identifiable (in any model), a relatively small number of predictors have to be responsible for

445 most of the effect on the outcome of interest; this is why such data structures were chosen in

446 this study.

447

448

\subsection{Conclusions}

449 In this research we have proposed a new method to conduct covariate selection for use with 450 relatively wide data. The method is based on a new approach to covariate selection stability 451 that incorporates a stability threshold to define which covariates should be included in a final 452 model. Results indicate the approach offers substantial reductions in covariate selection error rates compared with conventional model selection methods.

\section{Acknowledgements}

456 The author Eliana Lima is employed with the European Food Safety Authority (EFSA) in the

457 Unit ALPHA that provides scientific and administrative support to EFSA's Scientific

458 Activities in the area of animal health and welfare. However, the present article is published under the sole responsibility of the author Eliana Lima and may not be considered as an EFSA scientific output. The positions and opinions presented in this article are those of the

461 author alone and are not intended to represent any views/any official position or scientific

462 work of EFSA. To know about the views or scientific outputs of EFSA, please consult its 463 website under http://www.efsa.europa.eu. 


\section{References}

465 1. Wasserman, L. \& Roeder, K. High Dimensional Variable Selection. Ann. Stat. 1, $466 \quad 2178-2201(2009)$.

467 2. Sirimongkolkasem, T. \& Drikvandi, R. On Regularisation Methods for Analysis of High Dimensional Data. Ann. Data Sci. 6, 737-763 (2019).

3. Liu, J. Y., Zhong, W. \& Li, R. Z. A selective overview of feature screening for ultrahigh-dimensional data. Sci. China Math. 58, 2033-2054 (2015).

4. Statistical Learning with Sparsity: the Lasso and Generalizations. Available at: https://web.stanford.edu/ hastie/StatLearnSparsity/. (Accessed: 2nd March 2020)

5. Bogdan, M., Ghosh, J. K. \& Zak-Szatkowska, M. Selecting explanatory variables with the modified version of the bayesian information criterion. in Quality and Reliability Engineering International 24, 627-641 (2008).

6. Fan, J. \& Peng, H. Nonconcave penalized likelihood with a diverging number of parameters. Ann. Stat. 32, 928-961 (2004).

7. Tibshirani, R. Regression Shrinkage and Selection Via the Lasso. J. R. Stat. Soc. Ser. B 58, 267-288 (1996).

8. Zou, H. \& Hastie, T. Regularization and variable selection via the elastic net. $J . R$. Stat. Soc. Ser. B (Statistical Methodol. 67, 301-320 (2005).

482 9. Meinshausen, N. \& Bühlmann, P. Stability selection. J. R. Stat. Soc. Ser. B (Statistical 483 Methodol. 72, 417-473 (2010).

10. Baldassarre, L., Pontil, M. \& Mourão-miranda, J. Sparsity Is Better with Stability : Combining Accuracy and Stability for Model Selection in Brain Decoding. Front. Neurosci. 11, 62 (2017).

487 11. Heinze, G., Wallisch, C. \& Dunkler, D. Variable selection - A review and recommendations for the practicing statistician. Biometrical Journal 60, 431-449 
12. Alexander, D. H. \& Lange, K. Stability selection for genome-wide association. Genet. Epidemiol. 35, 722-728 (2011).

13. Kim, K., Koo, J. \& Sun, H. An empirical threshold of selection probability for analysis of high-dimensional correlated data. J. Stat. Comput. Simul. 90, 1606-1617 (2020).

14. Lima, E. et al. Use of bootstrapped, regularised regression to identify factors associated with lamb-derived revenue on commercial sheep farms. Prev. Vet. Med. 174, 104851 (2020).

15. Hyde, R. M., Green, M. J., Hudson, C. \& Down, P. M. Factors associated with daily weight gain in preweaned calves on dairy farms. Prev. Vet. Med. 190, 105320 (2021).

16. Spooner, A. et al. A comparison of machine learning methods for survival analysis of high-dimensional clinical data for dementia prediction. Sci. Rep. 10, 20410 (2020).

17. R Tibshirani, D. W. G. J. T. H. An Introduction to Statistical Learning with Applications in R (older version). Springer Texts in Statistics (New York : Springer, [2013] (2013, 2013).

18. Zhang, C. H. Nearly unbiased variable selection under minimax concave penalty. Ann. Stat. 38, 894-942 (2010).

19. Lima, E., Hyde, R. \& Green, M. Model selection for inferential models with high dimensional data: synthesis and graphical representation of multiple techniques. Sci. Rep. 11, 412 (2021).

509 20. Piotr Szulc. bigstep: Stepwise Selection for Large Data Sets. R package. (2019).

510 21. R Core Team. R: A language and environment for statistical computing (version 1.1.463). (2018).

512 22. Friedman, J., Hastie, T. \& Tibshirani, R. Regularization Paths for Generalized Linear Models via Coordinate Descent. J. Stat. Softw. 33, 1-22 (2010). 
514 23. Kuhn, M. et al. caret: Classification and Regression Training. (2019).

515

516

517

518

519

520

521

522

523

524

525

526

527

528

529

530

531

532

533

534

535

536

537

538

24. Breheny, P. \& Huang, J. Coordinate descent algorithms for nonconvex penalized regression, with applications to biological feature selection. Ann. Appl. Stat. 5, 232$253(2011)$

25. Breiman, L. Bagging Predictors. Mach. Learn. 24, 123-140 (1996).

26. Lima, E., Davies, P., Kaler, J., Lovatt, F. \& Green, M. Variable selection for inferential models with relatively high-dimensional data: Between method heterogeneity and covariate stability as adjuncts to robust selection. Sci. Rep. 10, 1-11 (2020).

27. Sauerbrei, W. The Use of Resampling Methods to Simplify Regression Models in Medical Statistics. J. R. Stat. Soc. Ser. C (Applied Stat. 48, 313-329 (1999).

28. Venables, W. N. \& Ripley, B. D. Modern applied statistics with S. Statistics and computing 45, (Springer-Verlag New York, 2002).

29. Nagelkerke, N. J. D. A note on a general definition of the coefficient of determination. Biometrika 78, 691-692 (1991).

30. Wang, F., Mukherjee, S., Richardson, S. \& Hill, S. M. High-dimensional regression in practice: an empirical study of finite-sample prediction, variable selection and ranking. Stat. Comput. 30, 697-719 (2020).

31. Pfeiffer, R. M., Redd, A. \& Carroll, R. J. On the impact of model selection on predictor identification and parameter inference. Comput. Stat. 32, 667-690 (2017).

32. Hofner, B., Boccuto, L. \& Göker, M. Controlling false discoveries in high-dimensional situations: Boosting with stability selection. BMC Bioinformatics 16, 144 (2015).

33. Dormann, C. F. et al. Collinearity: a review of methods to deal with it and a simulation study evaluating their performance. Ecography (Cop.). 36, 27-46 (2013).

34. Breiman, L. Random Forests. Mach. Learn. 45, 5-32 (2001). 
539 35. Kursa, M. B., Jankowski, A. \& Rudnicki, W. R. Boruta - A system for feature selection. Fundam. Informaticae 101, 271-285 (2010).

541 36. Lawlor, D. A., Tilling, K. \& Smith, G. D. Triangulation in aetiological epidemiology. Int. J. Epidemiol. 45, 1866-1886 (2016).

543 37. Munafò, M. R. \& Davey Smith, G. Robust research needs many lines of evidence. Nature 553, 399-401 (2018).

545

546 
547 Author contributions statement

548 EL, RH and MG contributed to development of statistical techniques and statistical analysis.

549 MG, RH and EL all contributed to the writing of the final manuscript. 


\begin{tabular}{|c|c|c|c|c|c|}
\hline Dataset name & $\mathbf{p}$ & $\begin{array}{c}\text { True } \\
\text { covariates }\end{array}$ & $\mathbf{n}$ & Description of correlation structure & $\mathbf{R}^{2}$ \\
\hline \multicolumn{6}{|c|}{ HS: CONTINUOUS NORMALLY DISTRIBUTED OUTCOME; HIGH SIGNAL } \\
\hline HS1 & 500 & 8 & 500 & all true and false variables uncorrelated & 0.70 \\
\hline HS2 & 500 & 8 & 500 & $\begin{array}{l}4 \text { true covariates correlated individually with } 10 \\
\text { false covariates at } 0.3\end{array}$ & 0.71 \\
\hline HS3 & 500 & 8 & 500 & $\begin{array}{l}4 \text { true covariates correlated individually with } 10 \\
\text { false covariates at } 0.5\end{array}$ & 0.69 \\
\hline HS4 & 500 & 8 & 500 & $\begin{array}{l}4 \text { true covariates correlated individually with } 10 \\
\text { false covariates at } 0.7\end{array}$ & 0.71 \\
\hline \multicolumn{6}{|c|}{ MS: CONTINUOUS NORMALLY DISTRIBUTED OUTCOME ; MEDIUM SIGNAL } \\
\hline MS1 & 500 & 8 & 500 & all true and false variables uncorrelated & 0.51 \\
\hline MS2 & 500 & 8 & 500 & $\begin{array}{l}4 \text { true covariates correlated individually with } 10 \\
\text { false covariates at } 0.3\end{array}$ & 0.46 \\
\hline MS3 & 500 & 8 & 500 & $\begin{array}{l}4 \text { true covariates correlated individually with } 10 \\
\text { false covariates at } 0.5\end{array}$ & 0.45 \\
\hline MS4 & 500 & 8 & 500 & $\begin{array}{l}4 \text { true covariates correlated individually with } 10 \\
\text { false covariates at } 0.7\end{array}$ & 0.45 \\
\hline \multicolumn{6}{|c|}{ LS: CONTINUOUS NORMALLY DISTRIBUTED OUTCOME; LOW SIGNAL } \\
\hline LS1 & 500 & 8 & 500 & all true and false variables uncorrelated & 0.29 \\
\hline LS2 & 500 & 8 & 500 & $\begin{array}{l}4 \text { true covariates correlated individually with } 10 \\
\text { false covariates at } 0.3\end{array}$ & 0.26 \\
\hline LS3 & 500 & 8 & 500 & $\begin{array}{l}4 \text { true covariates correlated individually with } 10 \\
\text { false covariates at } 0.5\end{array}$ & 0.28 \\
\hline LS4 & 500 & 8 & 500 & $\begin{array}{l}4 \text { true covariates correlated individually with } 10 \\
\text { false covariates at } 0.7\end{array}$ & 0.29 \\
\hline \multicolumn{6}{|c|}{ WID: CONTINUOUS NORMALLY DISTRIBUTED OUTCOME; WIDER DATASET } \\
\hline WID1 & 1000 & 8 & 500 & all true and false variables uncorrelated & 0.51 \\
\hline WID2 & 1000 & 8 & 500 & $\begin{array}{l}4 \text { true covariates correlated individually with } 10 \\
\text { false covariates at } 0.3\end{array}$ & 0.46 \\
\hline WID3 & 1000 & 8 & 500 & $\begin{array}{l}4 \text { true covariates correlated individually with } 10 \\
\text { false covariates at } 0.5\end{array}$ & 0.45 \\
\hline WID4 & 1000 & 8 & 500 & $\begin{array}{l}4 \text { true covariates correlated individually with } 10 \\
\text { false covariates at } 0.7\end{array}$ & 0.45 \\
\hline \multicolumn{6}{|c|}{ BIN: BINARY OUTCOME MEDIUM SIGNAL } \\
\hline BIN1 & 500 & 8 & 500 & all true and false variables uncorrelated & 0.48 \\
\hline BIN2 & 500 & 8 & 500 & $\begin{array}{l}4 \text { true covariates correlated individually with } 10 \\
\text { false covariates at } 0.3\end{array}$ & 0.48 \\
\hline BIN3 & 500 & 8 & 500 & $\begin{array}{l}4 \text { true covariates correlated individually with } 10 \\
\text { false covariates at } 0.5\end{array}$ & 0.47 \\
\hline BIN4 & 500 & 8 & 500 & $\begin{array}{l}4 \text { true covariates correlated individually with } 10 \\
\text { false covariates at } 0.7\end{array}$ & 0.47 \\
\hline \multicolumn{6}{|c|}{ IC: CONTINUOUS NORMALLY DISTRIBUTED OUTCOME; INCREASED COMPLEXITY } \\
\hline IC1 & 500 & 8 & 500 & $\begin{array}{l}4 \text { true covariates correlated with each other and } 20 \\
\text { false covariates at } 0.9\end{array}$ & 0.56 \\
\hline IC2 & 500 & 8 & 500 & $\begin{array}{l}4 \text { true covariates correlated with each other and } 92 \\
\text { false covariates at } 0.7\end{array}$ & 0.42 \\
\hline
\end{tabular}


560 Table 2. Results of full (non-bootstrapped) models conducted on 22 simulated datasets (Table

561 1) to illustrate the number of false positive and false negative covariates selected in final models 562 for each method.

563

\begin{tabular}{lcccccccc}
\hline & \multicolumn{1}{c}{ Model type } \\
Dataset name & \multicolumn{1}{c}{ sAIC } & \multicolumn{1}{c}{ mBIC } & \multicolumn{2}{c}{ enet } & \multicolumn{2}{c}{ MCP } \\
\hline HIGH SIGNAL & FP & FN & FP & FN & FP & FN & FP & FN \\
HS1 & & & & & & & & \\
HS2 & 11 & 1 & 0 & 1 & 22 & 0 & 1 & 1 \\
HS3 & 15 & 1 & 0 & 1 & 31 & 0 & 2 & 1 \\
HS4 & 15 & 1 & 0 & 1 & 31 & 0 & 3 & 1 \\
MEDIUM SIGNAL & 17 & 1 & 0 & 1 & 35 & 0 & 1 & 1 \\
MS1 & 14 & 1 & 0 & 1 & 9 & 0 & 7 & 1 \\
MS2 & 13 & 1 & 0 & 1 & 11 & 0 & 6 & 1 \\
MS3 & 25 & 1 & 0 & 1 & 27 & 0 & 15 & 1 \\
MS4 & 25 & 1 & 0 & 1 & 27 & 0 & 4 & 1 \\
LOW SIGNAL & & & & & & & & \\
LS1 & 15 & 1 & 0 & 3 & 38 & 0 & 17 & 1 \\
LS2 & 23 & 1 & 0 & 3 & 48 & 0 & 28 & 1 \\
LS3 & 19 & 1 & 0 & 2 & 52 & 0 & 14 & 1 \\
LS4 & 31 & 1 & 1 & 4 & 57 & 0 & 15 & 2 \\
WIDER DATASET & & & & & & & & \\
WID1 & 24 & 1 & 0 & 1 & 12 & 0 & 6 & 1 \\
WID2 & 24 & 1 & 0 & 1 & 19 & 0 & 1 & 1 \\
WID3 & 39 & 1 & 0 & 1 & 66 & 0 & 8 & 1 \\
WID4 & 46 & 1 & 0 & 1 & 45 & 0 & 8 & 1 \\
BINARY OUTCOME & & & & & & & \\
BIN1 & 17 & 1 & 0 & 1 & 20 & 0 & 5 & 1 \\
BIN2 & 17 & 1 & 0 & 1 & 33 & 0 & 8 & 1 \\
BIN3 & 28 & 1 & 0 & 1 & 94 & 0 & 9 & 1 \\
BIN4 & 20 & 2 & 1 & 2 & 43 & 0 & 8 & 2 \\
INCREASED COMPLEXITY & & & & & & \\
IC1 & 15 & 1 & 0 & 2 & 30 & 0 & 24 & 2 \\
IC2 & 25 & 2 & 0 & 3 & 51 & 0 & 14 & 2
\end{tabular}

565

566

567 Key; sAIC - covariate selection based on stepwise Akaike information criterion, mBIC - covariate selection

568 based on a stepwise on a modified Bayesian Information Criterion, enet - covariate section based on elastic net

569

570

571

572

573 
Table 3. Results of models incorporating covariate selection stability (with selection threshold 575 set at $\mathrm{x}=0$ ), conducted on 22 simulated datasets (Table 1), to illustrate the number of false 576 positive and false negative covariates selected in final models for each method.

\begin{tabular}{lcccccccccc}
\multicolumn{110}{c}{} & \multicolumn{10}{c}{ Model type } \\
\multicolumn{1}{c}{} & \multicolumn{1}{c}{ sAIC } & \multicolumn{1}{c}{ mBIC } & \multicolumn{1}{c}{ enet } & \multicolumn{2}{c}{ MCP } & \multicolumn{2}{c}{ Combi } \\
Dataset name & FP & FN & FP & FN & FP & FN & FP & FN & FP & FN \\
\hline HIGH SIGNAL & & & & & & & & & & \\
HS1 & 0 & 0 & 0 & 0 & 0 & 0 & 0 & 0 & 0 & 0 \\
HS2 & 0 & 0 & 0 & 0 & 0 & 0 & 0 & 0 & 0 & 0 \\
HS3 & 0 & 0 & 0 & 0 & 0 & 0 & 0 & 0 & 0 & 0 \\
HS4 & 0 & 0 & 1 & 0 & 0 & 0 & 0 & 0 & 0 & 0 \\
MEDIUM SIGNAL & & & & & & & & & \\
MS1 & 0 & 0 & 0 & 0 & 0 & 0 & 0 & 0 & 0 & 0 \\
MS2 & 0 & 0 & 0 & 0 & 0 & 0 & 0 & 0 & 0 & 0 \\
MS3 & 0 & 0 & 0 & 0 & 0 & 0 & 0 & 0 & 0 & 0 \\
MS4 & 1 & 0 & 0 & 0 & 0 & 0 & 0 & 0 & 0 & 0 \\
LOW SIGNAL & & & & & & & & & & \\
LS1 & 0 & 2 & 0 & 1 & 0 & 2 & 0 & 1 & 0 & 0 \\
LS2 & 0 & 2 & 1 & 0 & 0 & 2 & 0 & 2 & 0 & 0 \\
LS3 & 0 & 3 & 2 & 0 & 0 & 2 & 0 & 1 & 1 & 0 \\
LS4 & 1 & 1 & 1 & 1 & 0 & 2 & 0 & 1 & 0 & 1 \\
WIDER DATASET & & & & & & & & & \\
WID1 & 0 & 0 & 0 & 0 & 0 & 0 & 0 & 0 & 0 & 0 \\
WID2 & 0 & 0 & 0 & 0 & 0 & 0 & 0 & 0 & 0 & 0 \\
WID3 & 0 & 0 & 0 & 0 & 0 & 0 & 0 & 0 & 0 & 0 \\
WID4 & 0 & 0 & 0 & 0 & 0 & 0 & 0 & 0 & 0 & 0 \\
BINARY OUTCOME & & & & & & & & & \\
BIN1 & 0 & 0 & 2 & 0 & 0 & 0 & 0 & 0 & 0 & 0 \\
BIN2 & 0 & 1 & 1 & 0 & 0 & 0 & 0 & 0 & 0 & 0 \\
BIN3 & 1 & 0 & 1 & 0 & 0 & 0 & 0 & 0 & 1 & 0 \\
BIN4 & 0 & 1 & 1 & 1 & 0 & 1 & 0 & 1 & 0 & 1 \\
INCREASED COMPLEXITY & & & & & & & \\
IC1 & 0 & 4 & 1 & 0 & 0 & 2 & 0 & 3 & 0 & 3 \\
IC2 & 0 & 3 & 0 & 2 & 0 & 3 & 0 & 4 & 0 & 3
\end{tabular}

581 Key; sAIC - covariate selection based on stepwise Akaike information criterion, mBIC - covariate selection 582 based on a stepwise on a modified Bayesian Information Criterion, enet - covariate section based on elastic net 583 regression, MCP - covariate selection based on minimax convex penalty regression, Combi - covariate

584 selection based on a synthesised combination of sAIC, mBIC, enet and MCP, FP - number of false positive 585 covariates selected, FN - number of false negative variables selected 
Figure 1.

590
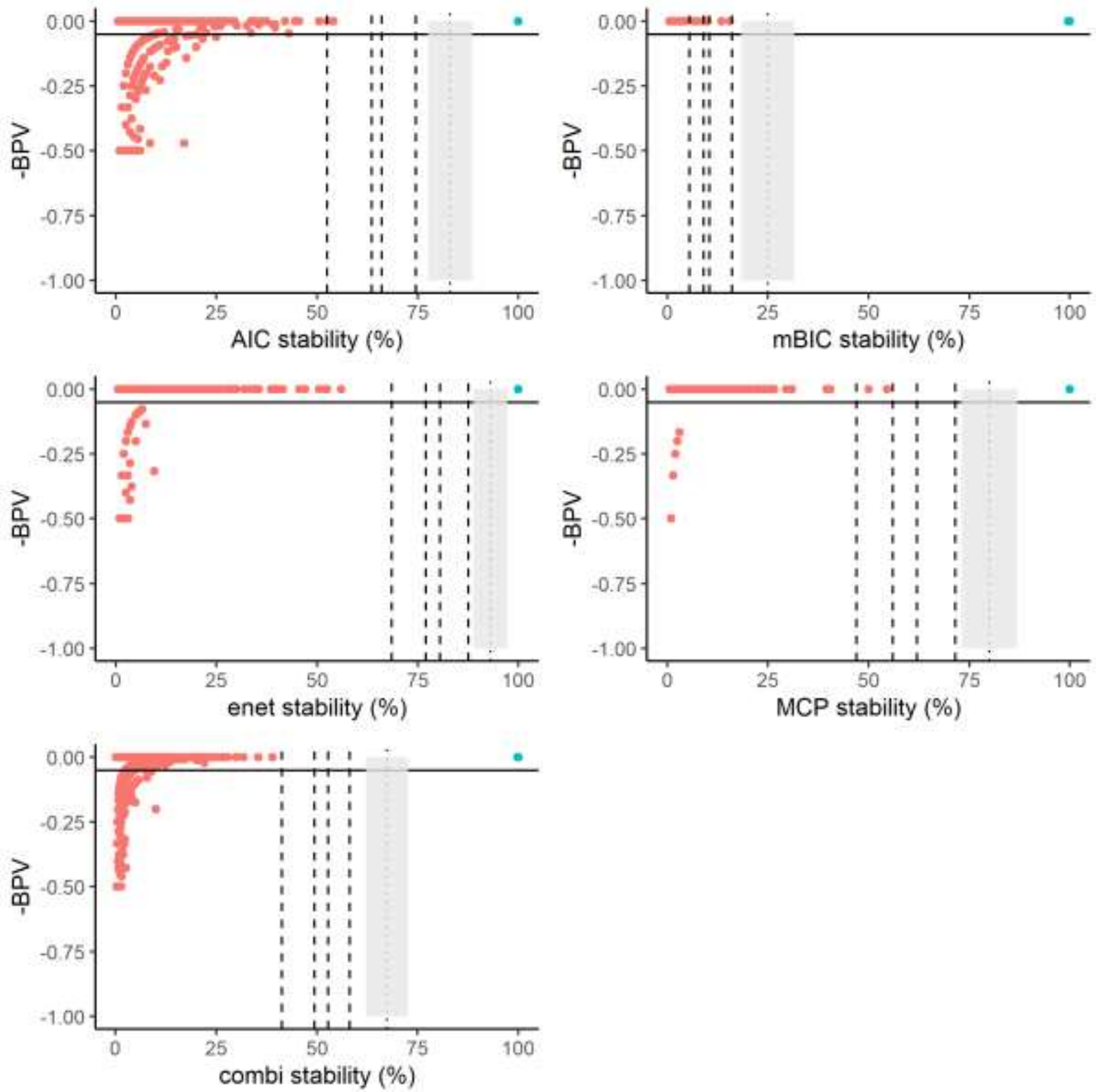
Figure 2.

605

606
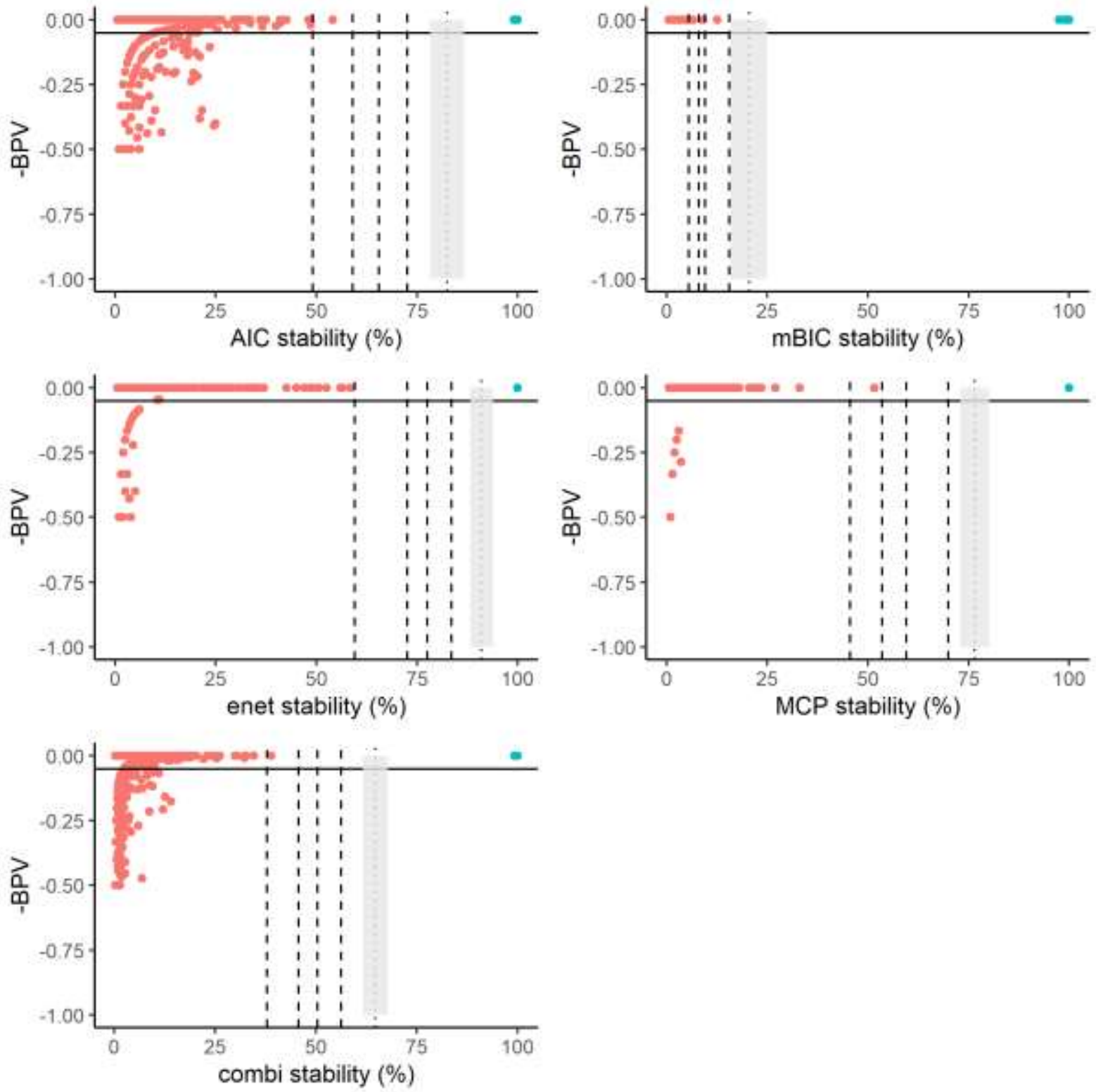

607

608

609

610

611

612

613

614 
615

616
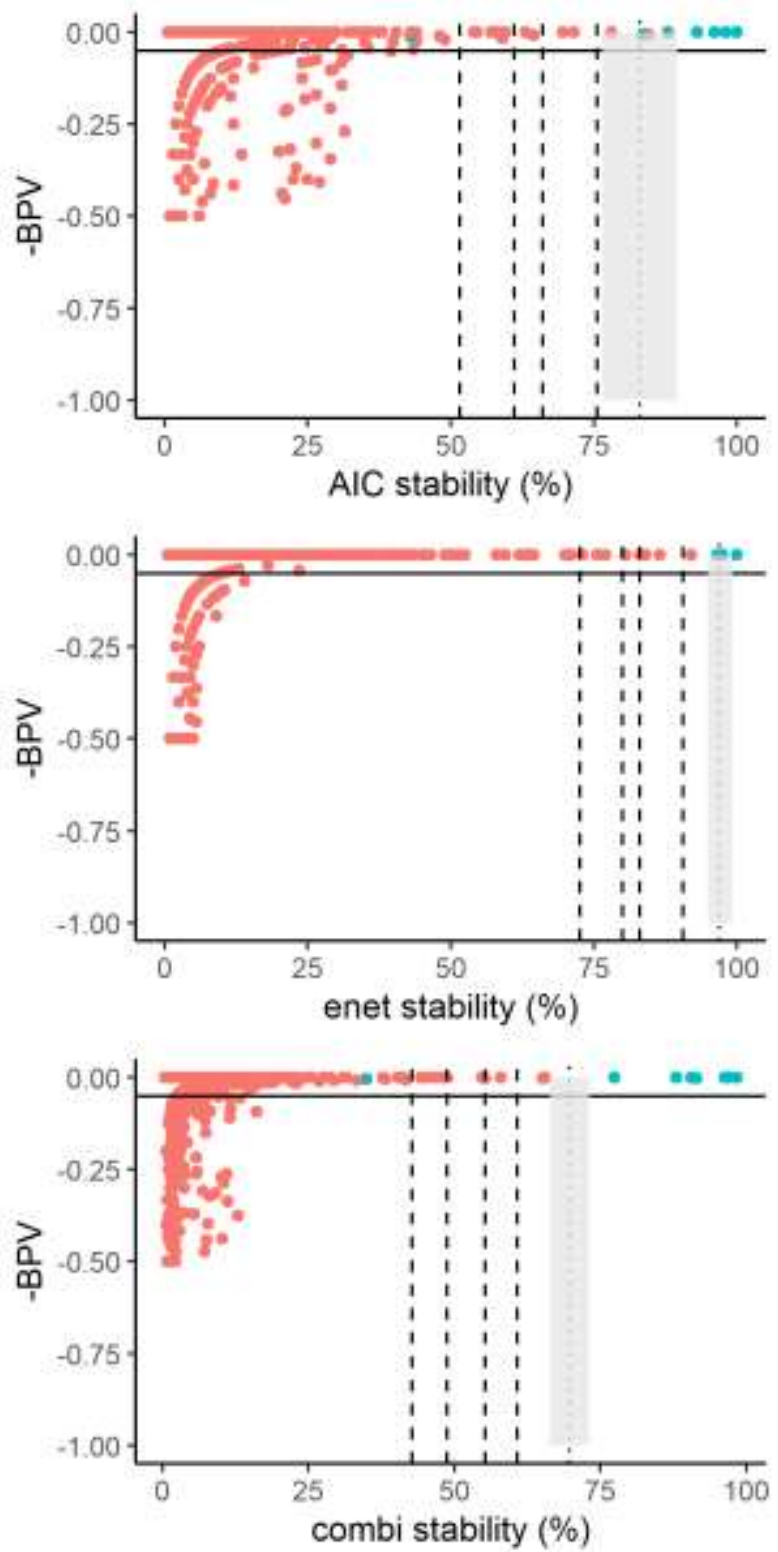

617

618

619
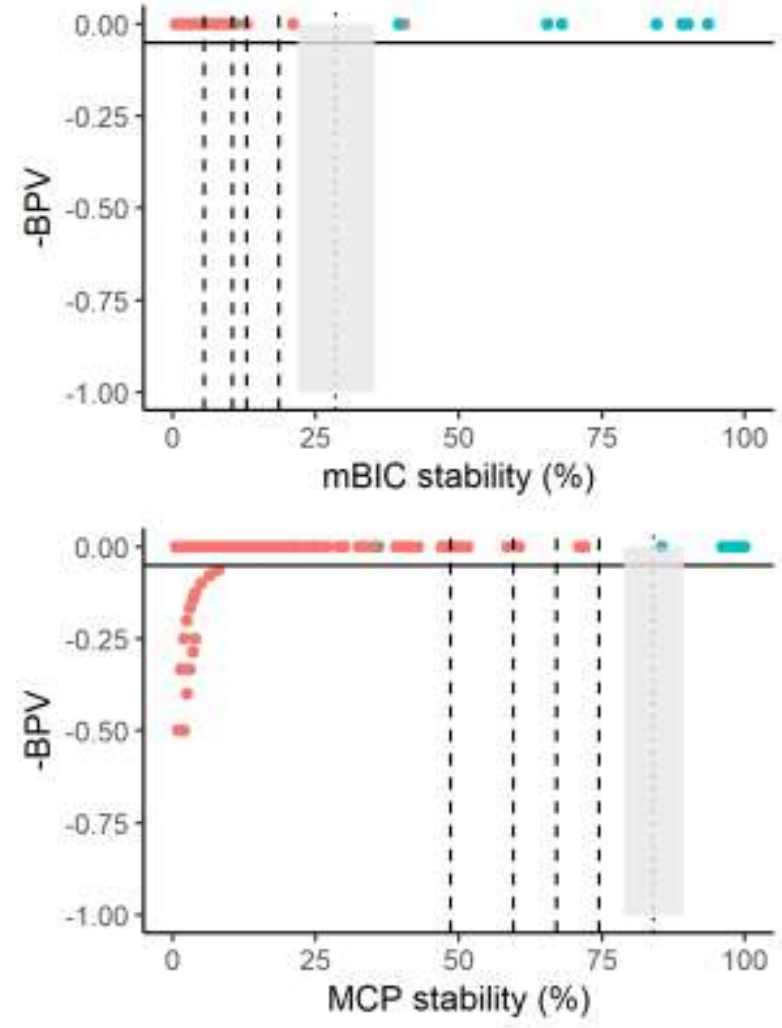


\section{Supplementary Files}

This is a list of supplementary files associated with this preprint. Click to download.

- Supplementarymaterials.docx 